\title{
Parabolic Partial Differential Equations with Border Conditions of Dirichlet as Inverse Moments Problem
}

\author{
Mara B. Pintarelli1,2 \\ ${ }^{1}$ Grupo de Aplicaciones Matematicas y Estadisticas de la Facultad de Ingenieria (GAMEFI), Universidad Nacional de La Plata, \\ Buenos Aires, Argentina \\ ${ }^{2}$ Departamento de Matematica, Facultad de Ciencias Exactas, Universidad Nacional de La Plata, Buenos Aires, Argentina \\ Email: mariabpintarelli@gmail.com
}

How to cite this paper: Pintarelli, M.B. (2017) Parabolic Partial Differential Equations with Border Conditions of Dirichlet as Inverse Moments Problem. Applied Mathematics, 8, 15-25.

http://dx.doi.org/10.4236/am.2017.81002

Received: December 7, 2016

Accepted: January 15, 2017

Published: January 18, 2017

Copyright $\odot 2017$ by author and Scientific Research Publishing Inc. This work is licensed under the Creative Commons Attribution International License (CC BY 4.0).

http://creativecommons.org/licenses/by/4.0/ (c) (i) Open Access

\begin{abstract}
We considerer parabolic partial differential equations: $w_{t}-\left(w_{x}\right)_{x}=r(x, t)$ under the conditions $w\left(a_{1}, t\right)=k_{1}(t) \quad w\left(b_{1}, t\right)=k_{2}(t), w\left(x, a_{2}\right)=h_{1}(t)$ on a region $E=\left(a_{1}, b_{1}\right) \times\left(a_{2}, b_{2}\right) ; b_{2}=\infty$. We will see that an approximate solution can be found using the techniques of generalized inverse moments problem and also bounds for the error of estimated solution. First we transform the parabolic partial differential equation to the integral equation
\end{abstract} $\int_{a_{1}}^{b_{1}} \int_{a_{2}}^{b_{2}} e^{-m(x+t)}\left(w_{x}(x, t)-w_{t}(x, t)\right) \mathrm{d} t \mathrm{~d} x=\varphi_{1}(m)$. Using the inverse moments problem techniques we obtain an approximate solution $p_{n}(x, t)$ of $w_{x}(x, t)-w_{t}(x, t)$. Then we find a numerical approximation of $w(x, t)$ when solving the integral equation

$\int_{a_{1}}^{b_{1}} \int_{a_{2}}^{b_{2}} e^{-(m+1)(x+1)-(z+1)(t+1)}(w(x, t)(t-x)) \mathrm{d} t \mathrm{~d} x=\varphi_{2}(m, z)$, because solving the previous integral equation is equivalent to solving the equation $w_{x}(x, t)-w_{t}(x, t)=p_{n}(x, t)$.

\section{Keywords}

Parabolic PDEs, Integral Equations, Generalized Moment Problem

\section{Introduction}

We considerer parabolic partial differential equation of the form:

$$
w_{t}-\left(w_{x}\right)_{x}=r(x, t)
$$


where the unknown function $w(x, t)$ is defined in $E=\left(a_{1}, b_{1}\right) \times\left(a_{2}, \infty\right)$ and $r(x, t)$ is known function. Under the conditions

$$
\begin{gathered}
w\left(a_{1}, t\right)=k_{1}(t) \quad w\left(b_{1}, t\right)=k_{2}(t) \\
w\left(x, a_{2}\right)=h_{1}(t)
\end{gathered}
$$

This problem was studied under conditions of Cauchy in [1] and under conditions of Neumann in [2].

Parabolic differential equations are commonly used in the fields of engineering and science for simulating physical processes. These equations describe various processes in viscous fluid flow, filtration of liquids, gas dynamics, heat conduction, elasticity, biological species, chemical reactions, environmental pollution, etc. [3] [4].

In a variety of cases, approximations are used to convert parabolic PDEs to ordinary differential equations or even to algebraic equations. The existence and uniqueness properties of this problem are presented in literature. Several numerical methods have been proposed for the solution of this problem [5] [6] [7].

Parabolic partial differential equations have been numerically solved by using a variety of techniques [8] [9] [10] [11].

The finite element method for the numerical solution of partial differential equations is a general method covering all the three main types of equations: elliptic, parabolic and hyperbolic equations [12].

Some meshless schemes to solve differential partial equations are the diffuse element method [13], the partition of unity method [14], the element-free Galerkin method [15], the reproducing kernel particle method [16], the finite point method [17], the meshless local Petrov-Galerkin method [18], the use of radial basis functions [19] and the general finite difference method [20].

The d-dimensional generalized moment problem [21] [22] [23] [24] [25] can be posed as follows: find a function $f$ on a domain $\Omega \subset \boldsymbol{R}^{d}$ satisfying the sequence of equations

$$
\int_{\Omega} f(x) g_{i}(x) \mathrm{d} x=\mu_{i} \quad i \in N
$$

where $\left(g_{i}\right)$ is a given sequence of functions lying in $\mathbf{L}^{2}(\Omega)$ linearly independent, and the sequence of real numbers $\left\{\mu_{i}\right\}_{i \in N}$ is the known data.

The moments problem of Hausdorff is a classic example of moments problem, and is to find a function $f(x)$ in $(a, b)$ such that

$$
\mu_{i}=\int_{a}^{b} x^{i} f(x) \mathrm{d} x \quad i \in N
$$

In this case the functions $g_{i}(x)=x^{i} i \epsilon N$. If the interval of integration is $(0, \infty)$ we have the problem of moments of Stieltjes; if the interval of integration is $(-\infty, \infty)$ we have the problem of moments of Hamburger.

Moment problem is usually ill-posed in the sense that there may be no solution and if there is no continuous dependence on the given data. There are various methods of constructing regularized solutions, that is, approximate solutions stable with respect to the given data. One of them is the method of truncated expansion. 
The method of truncated expansion consists in approximating (4) by finite moment problems

$$
\int_{\Omega} f(x) g_{i}(x) \mathrm{d} x=\mu_{i} \quad i=1,2, \cdots, n
$$

and consider as an approximate solution of $f(x)$ to $p_{n}(x)=\sum_{i=0}^{n} \lambda_{i} \varphi_{i}(x)$. The $\varphi_{i}(x)$ result from orthonormalize $\left\langle g_{1}, g_{2}, \cdots, g_{n}\right\rangle$ and $\lambda_{i}$ are coefficients as a function of the $\mu_{i}$.

Solved in the subspace $\left\langle g_{1}, g_{2}, \cdots, g_{n}\right\rangle$ generated by $g_{1}, g_{2}, \cdots, g_{n}$ (5) is stable. Considering the case where the data $\mu=\left(\mu_{1}, \mu_{2}, \cdots, \mu_{n}\right)$ are inexact, convergence theorems and error estimates for the regularized solutions they are applied.

In this paper we consider a different way to numerically solve the problem given by Equation (1) with conditions (2) and (3): we first transform it into an integral equation which we then handle as a bidimensional moment problem. This approach was already suggested by Ang [25] in relation with the heat conduction equation.

The work is organized as follows: in Section 2 first we transform the parabolic partial differential equation to the integral equation

$$
\int_{a_{1}}^{b_{1}} \int_{a_{2}}^{b_{2}} e^{-m(x+t)}\left(w_{x}(x, t)-w_{t}(x, t)\right) \mathrm{d} t \mathrm{~d} x=\varphi_{1}(m)
$$

Using the inverse moments problem techniques we obtain an approximate solution $p_{n}(x, t)$ of $w_{x}(x, t)-w_{t}(x, t)$. Then we find a numerical approximation of $w(x, t)$ when solving the integral equation

$$
\int_{a_{1}}^{b_{1}} \int_{a_{2}}^{b_{2}} e^{-(m+1)(x+1)-(z+1)(t+1)}(w(x, t)(t-x)) \mathrm{d} t \mathrm{~d} x=\varphi_{2}(m, z)
$$

In Section 3 the method is illustrated with examples.

\section{Resolution of the Parabolic Partial Differential Equations}

Let $F(w(x, t))=r(x, t)$ be a partial differential equations such as (1). The solution $w(x, t)$ is defined on the region $E=\left(a_{1}, b_{1}\right) \times\left(a_{2}, b_{2}\right), b_{2}=\infty$ and verifies on the boundary $C=\partial E$ :

$$
\begin{gathered}
w\left(a_{1}, t\right)=k_{1}(t) \quad w\left(b_{1}, t\right)=k_{2}(t) \\
w\left(x, a_{2}\right)=h_{1}(t)
\end{gathered}
$$

We apply the technique used in [2]. Let $F^{*}=\left(F_{1}(w), F_{2}(w)\right)$ be a vectorial field such that $w$ verifies $\operatorname{div}\left(F^{*}\right)=h^{*}(w)$ with $h^{*}$ a known function and, reciprocally, if $w$ verifies $\operatorname{div}\left(F^{*}\right)=h^{*}(w)$ then $F(w(x, t))=r(x, t)$.

Specifically in this case $F(w(x, t))=w_{t}-\left(w_{x}\right)_{x}$ and we take

$$
\begin{gathered}
F^{*}=\left(F_{1}(w), F_{2}(w)\right)=\left(w_{x},-w\right) \\
h^{*}(w)=-r(x, t)
\end{gathered}
$$

Let $u(m, z, x, t)$ be the auxiliary function

$$
u(m, z, x, t)=e^{-(m+1) x-(z+1) t}
$$


Since

$$
u \operatorname{div}\left(F^{*}\right)=u h^{*}(w)
$$

we have

$$
\iint_{E} u \operatorname{div}\left(F^{*}\right) \mathrm{d} A=\iint_{E} u h^{*}(w) \mathrm{d} A
$$

Moreover, as

$$
\begin{gathered}
u \operatorname{div}\left(F^{*}\right)=\operatorname{div}\left(u F^{*}\right)-F^{*} \cdot \nabla u \\
\iint_{E} u \operatorname{div}\left(F^{*}\right) \mathrm{d} A=\iint_{E} \operatorname{div}\left(u F^{*}\right) \mathrm{d} A-\iint_{E} F^{*} \nabla u \mathrm{~d} A
\end{gathered}
$$

where $\nabla u=\left(u_{x}, u_{t}\right)$ besides

$$
\begin{aligned}
\iint_{E} \operatorname{div}\left(u F^{*}\right) \mathrm{d} A & =\iint_{E}\left(\left(u w_{x}\right)_{x}-(u w)_{t}\right) \mathrm{d} A= \\
& =\iint_{E} u \operatorname{div}\left(F^{*}\right) \mathrm{d} A+\iint_{E}\left(\left(u_{x} w_{x}\right)-\left(u_{t} w\right)\right) \mathrm{d} A
\end{aligned}
$$

Then of (6) and (7)

$$
\iint_{E}\left(u_{x} w_{x}-u_{t} w\right) \mathrm{d} A=\iint_{E} F^{*} \nabla u \mathrm{~d} A
$$

On the other hand it can be proved that, after several calculations, (8) is written as

$\int_{a_{1}}^{b_{1}} w\left(x, a_{2}\right) u\left(m, z, x, a_{2}\right) \mathrm{d} x+\frac{z+1}{m+1} \int_{a_{2}}^{b_{2}}\left(w\left(b_{1}, t\right) u\left(m, z, b_{1}, t\right)-w\left(a_{1}, t\right) u\left(m, z, a_{1}, t\right)\right) \mathrm{d} t$ $=\frac{z+1}{m+1} \int_{a_{1}}^{b_{1}} \int_{a_{2}}^{b_{2}} w_{x}(x, t) u \mathrm{~d} t \mathrm{~d} x-\int_{a_{1}}^{b_{1}} \int_{a_{2}}^{b_{2}} w_{t}(x, t) u \mathrm{~d} t \mathrm{~d} x$

and if $z=m$ then

$$
\begin{aligned}
& \int_{a_{1}}^{b_{1}} \int_{a_{2}}^{b_{2}}\left(w_{x}(x, t)-w_{t}(x, t)\right) u(m, m, x, t) \mathrm{d} t \mathrm{~d} x \\
& =\int_{a_{1}}^{b_{1}} w\left(x, a_{2}\right) u\left(m, m, x, a_{2}\right) \mathrm{d} x \\
& \quad+\int_{a_{2}}^{b_{2}}\left(w\left(b_{1}, t\right) u\left(m, m, b_{1}, t\right)-w\left(a_{1}, t\right) u\left(m, m, a_{1}, t\right)\right) \mathrm{d} t=\varphi_{1}(m)
\end{aligned}
$$

We take a base $\left\{\psi_{i}(m)\right\}_{i}$ of $L^{2}\left(a_{2}, b_{2}\right)$ and then the above equation can be transformed into a generalized moment problem

$$
\int_{a_{1}}^{b_{1}} \int_{a_{2}}^{b_{2}}\left(w_{x}(x, t)-w_{t}(x, t)\right) H_{i}(x, t) \mathrm{d} t \mathrm{~d} x=\mu_{i}
$$

where

$$
H_{i}(x, t)=\int_{a_{2}}^{b_{2}} u(m, n, x, t) \psi_{i}(m) \mathrm{d} m
$$

and

$$
\mu_{i}=\int_{a_{2}}^{b_{2}} \varphi_{1}(m) \psi_{i}(m) \mathrm{d} m
$$

We can apply the truncated expansion method detailed in [24] and generalized in [25] [26] to find an approximation $p_{n}(x, t)$ for $w_{x}(x, t)-w_{t}(x, t)$ for the corresponding finite problem with $i=0,1, \cdots, n$ where $n$ is the number of moments $\mu_{i}$. We consider the base $\phi_{i}(x, t) i=0,1,2, \cdots$ obtained by applying the Gram-Schmidt orthonormalization process on $H_{i}(x, t) i=0,1,2, \cdots$ and add- 
ing to the resulting set the necessary functions until reaching an orthonormal basis.

We approach the solution $w_{x}(x, t)-w_{t}(x, t)$ with [25] [26]:

$$
p_{n}(x, t)=\sum_{i=0}^{n} \lambda_{i} \phi_{i}(x, t) \text { where } \lambda_{i}=\sum_{j=0}^{i} C_{i j} \mu_{j} \quad i=0,1,2, \cdots, n
$$

And the coefficients $C_{i j}$ verifies

$$
C_{i j}=\left(\sum_{k=j}^{i-1}(-1) \frac{\left\langle H_{i}(x, t) \mid \phi_{k}(x, t)\right\rangle}{\left\|\phi_{k}(x, t)\right\|^{2}} C_{k j}\right) \cdot\left\|\phi_{i}(x, t)\right\|^{-1} \quad 1<i \leq n ; 1 \leq j<i
$$

The terms of the diagonal are

$$
C_{i i}=\left\|\phi_{i}(x, t)\right\|^{-1} \quad i=0,1, \cdots, n .
$$

The proof of the following theorem is in [27] [28]. In [28] he proof is done for $b_{2}$ finite. If $b_{2}=\infty$ instead of taking polynomials the Legendre are taken polynomials of Laguerre. In [2] the demonstration is done for the one-dimensional case.

Theorem. Let $\left\{\mu_{i}\right\}_{i=0}^{n}$ be a set of real numbers and suppose that $f(x, t)$ verify for some $\varepsilon$ and $E$ (two positive numbers)

$$
\begin{gathered}
\sum_{i=0}^{n}\left|\int_{a_{2}}^{b_{2}} \int_{a_{1}}^{b_{1}} H_{i}(x, t) f(x, t) \mathrm{d} x \mathrm{~d} t-\mu_{i}\right|^{2} \leq \varepsilon^{2} \\
\int_{a_{2}}^{b_{2}} \int_{a_{1}}^{b_{1}}\left(x f_{x}^{2}+t f_{t}^{2}\right) e^{x+t} \mathrm{~d} x \mathrm{~d} t \leq E^{2}
\end{gathered}
$$

then

$$
\int_{a_{2}}^{b_{2}} \int_{a_{1}}^{b_{1}}|f(x, t)|^{2} \mathrm{~d} x \mathrm{~d} t \leq \min _{i}\left\{\left\|C C^{\mathrm{T}}\right\| \varepsilon^{2}+\frac{E^{2}}{8(i+1)^{2}} ; i=0,1, \cdots, n\right\}
$$

where $C$ is the triangular matrix with elements $C_{i j} \quad(1<i \leq n ; 1 \leq j<i)$, and

$$
\int_{a_{2}}^{b_{2}} \int_{a_{1}}^{b_{1}}\left|p_{n}(x, t)-f(x, t)\right|^{2} \mathrm{~d} x \mathrm{~d} t \leq\left\|C C^{\mathrm{T}}\right\| \varepsilon^{2}+\frac{E^{2}}{8(n+1)^{2}}
$$

It must be fulfilled that

$$
t^{i} f(x, t) \rightarrow 0 \quad \text { if } \quad t \rightarrow \infty \quad \forall i \epsilon N
$$

If we apply the truncated expansion method to solve Equation (9) we obtain an approximation $p_{n}(x, t)$ for $f(x, t)=w_{x}(x, t)-w_{t}(x, t)$. Then we have an equation in first order partial derivatives of the form

$$
w_{x}(x, t)-w_{t}(x, t)=p_{n}(x, t)
$$

It is solved as in [28], we can prove that solving this equation is equivalent to solving the integral equation

$$
\int_{a_{1}}^{b_{1}} \int_{a_{2}}^{b_{2}} u(m, z, x, t) w(x, t)(t-x) \mathrm{d} t \mathrm{~d} x=\varphi_{2}(m, z)
$$

where

$$
\begin{aligned}
\varphi_{2}(m, z)= & \int_{a_{2}}^{b_{2}}\left[u\left(m, z, b_{1}, t\right) w\left(b_{1}, t\right)-u\left(m, n, a_{1}, t\right) w\left(a_{1}, t\right)\right] \mathrm{d} t+ \\
& +\int_{a_{1}}^{b_{1}}\left[u\left(m, z, x, b_{2}\right) w\left(x, b_{2}\right)-u\left(m, n, x, a_{2}\right) w\left(x, a_{2}\right)\right] \mathrm{d} x-\int_{a_{1}}^{b_{1}} \int_{a_{2}}^{b_{2}} u p_{n} \mathrm{~d} t \mathrm{~d} x
\end{aligned}
$$


and $u(m, z, x, t)=e^{-(m+1)(x+1)-(z+1)(t+1)}$. Again we take a base $\left\{\psi_{i j}^{*}(m, z)\right\}_{i j}$ of $L^{2}(E)$ and then the above equation can be transformed into a generalized moment problem

$$
\int_{a_{1}}^{b_{1}} \int_{a_{2}}^{b_{2}} w(x, t) H_{i j}^{*}(x, t) \mathrm{d} t \mathrm{~d} x=\mu_{i j}^{*}
$$

where

$$
H_{i j}^{*}(x, t)=\int_{a_{1}}^{b_{1}} \int_{a_{2}}^{b_{2}} u(m, n, x, t)(t-x) \psi_{i j}^{*}(m, z) \mathrm{d} z \mathrm{~d} m
$$

and

$$
\mu_{i j}^{*}=\int_{a_{1}}^{b_{1}} \int_{a_{2}}^{b_{2}} \varphi_{2}(m, z) \psi_{i j}^{*}(m, z) \mathrm{d} z \mathrm{~d} m
$$

Applying again the techniques of generalized moments problem we found an approximate solution for $w(x, t)$.

\section{Numerical Examples}

\subsection{Example 1}

We consider the equation

$$
w_{t}=w_{x x}-\frac{1+e^{-x-t}\left(1+t^{2}\left(2+x e^{x}\right)\right)}{1+t^{2}} \text { in } E=(0,1) \times(0, \infty)
$$

and conditions

$$
w(0, t)=e^{-t} ; \quad w(1, t)=\left(e^{-1}+1\right) e^{-t} ; \quad w(x, 0)=x+e^{-x}
$$

The solution is $w(x, t)=e^{-t}\left(e^{-x}+x\right)$

First step: approximates $f(x, t)=w_{x}(x, t)-w_{t}(x, t)$

We take the base $\psi_{i}(m)=m^{i-1} e^{-m} \quad i=1,2, \cdots, 5$

Accuracy is $\left(\int_{a_{1}}^{b_{1}} \int_{a_{2}}^{b_{2}}\left|f(x, t)-p_{5}(x, t)\right|^{2} \mathrm{~d} t \mathrm{~d} x\right)^{\frac{1}{2}}=0.409962$.

In Figure 1(a) the exact solution and the approximate solution are compared Second step: approximates $w(x, t)$

We take the base $\psi_{i j}(m, z)=m^{i-1} z^{j-1} e^{-m-z} \quad i=1,2,3 \quad j=1,2,3$

Accuracy is $\left(\int_{a_{1}}^{b_{1}} \int_{a_{2}}^{b_{2}}\left|w(x, t)-p_{9}(x, t)\right|^{2} \mathrm{~d} t \mathrm{~d} x\right)^{\frac{1}{2}}=0.131406$.

In Figure 1(b) the exact solution and the approximate solution are compared.

\subsection{Example 2}

We consider the equation

$$
w_{t}=w_{x x} \quad \text { in } \quad E=(0,1) \times(0, \infty)
$$

and conditions

$$
w(0, t)=\operatorname{Exp}\left(-\frac{\pi^{2}}{4} t\right) ; w(1, t)=0 ; \quad w(x, 0)=\operatorname{Cos}\left(\frac{\pi}{2} x\right)
$$




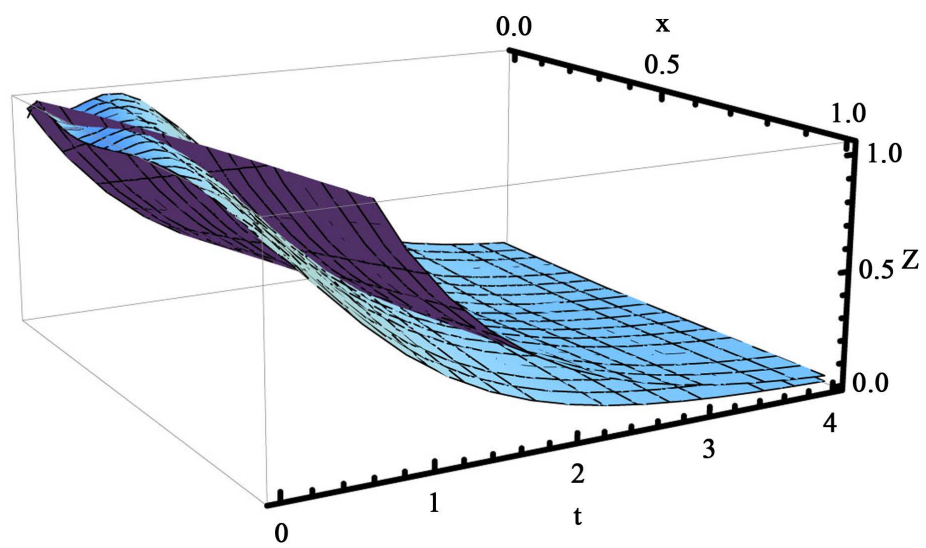

(a)

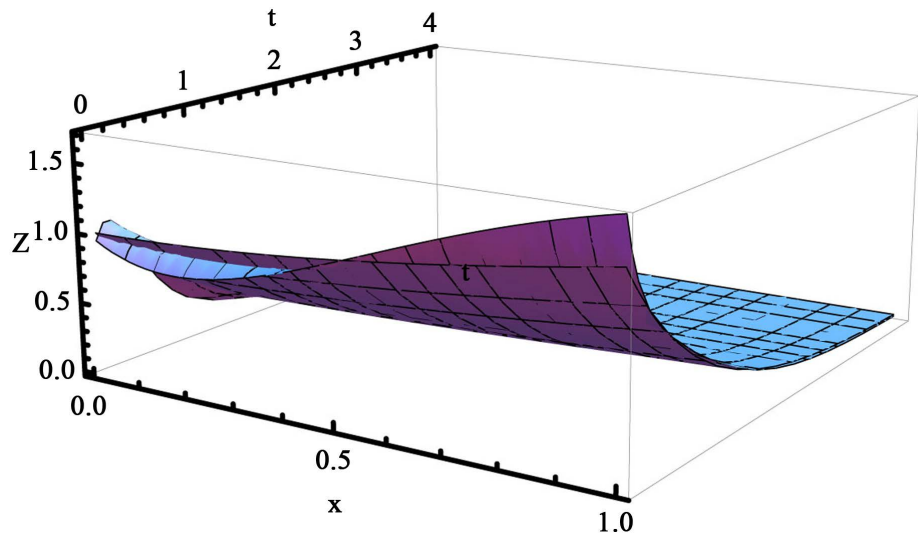

(b)

Figure 1. (a) $w_{x}(x, t)-w_{t}(x, t)$ and $p_{5}(x, t) ;(b) w(x, t)$ and $p_{9}(x, t)$.

The solution is $w(x, t)=\operatorname{Exp}\left(-\frac{\pi^{2}}{4} t\right) \operatorname{Cos}\left(\frac{\pi}{2} x\right)$

First step: approximates $f(x, t)=w_{x}(x, t)-w_{t}(x, t)$

We take the base $\psi_{i}(m)=m^{i-1} e^{-m} \quad i=1,2, \cdots, 5$

Accuracy is $\left(\int_{a_{1}}^{b_{1}} \int_{a_{2}}^{b_{2}}\left|f(x, t)-p_{5}(x, t)\right|^{2} \mathrm{~d} t \mathrm{~d} x\right)^{\frac{1}{2}}=0.294629$.

In Figure 2(a) the exact solution and the approximate solution are compared Second step: approximates $w(x, t)$

We take the base $\psi_{i j}(m, z)=m^{i-1} z^{j-1} e^{-m-z} \quad i=1,2,3 \quad j=1,2,3$

Accuracy is $\left(\int_{a_{1}}^{b_{1}} \int_{a_{2}}^{b_{2}}\left|w(x, t)-p_{9}(x, t)\right|^{2} \mathrm{~d} t \mathrm{~d} x\right)^{\frac{1}{2}}=0.0673978$.

In Figure 2(b) the exact solution and the approximate solution are compared.

\subsection{Example 3}

We consider the equation

$$
w_{x x}-w_{t}=p(t) w_{x}+e^{-x-3 t}(5+t) \text { in } E=(0,1) \times(0, \infty)
$$

where $p(t)$ is unknown. And conditions 


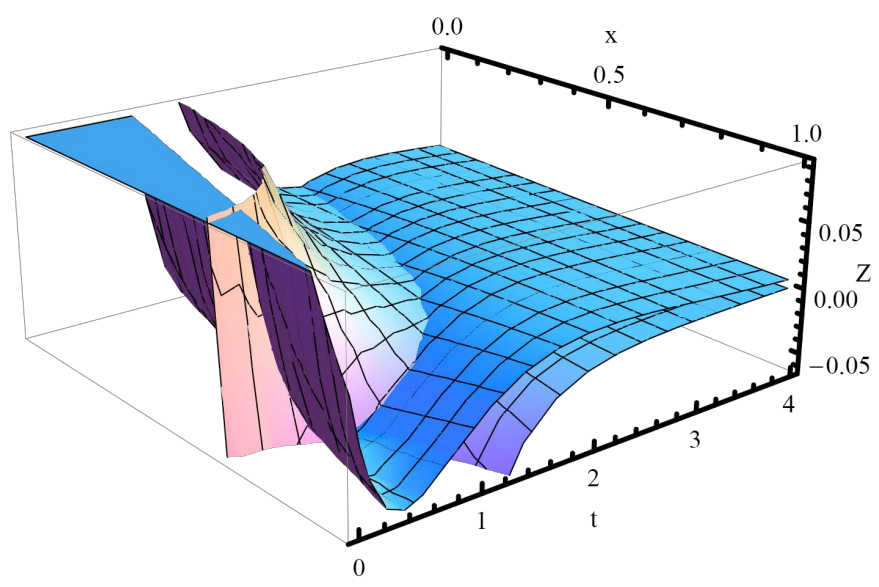

(a)

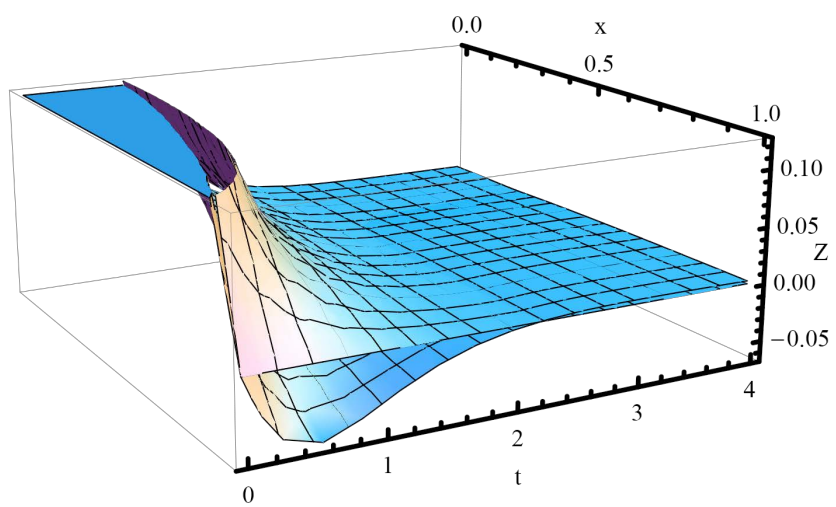

(b)

Figure 2. (a) $w_{x}(x, t)-w_{t}(x, t)$ and $p_{5}(x, t) ;(\mathrm{b}) \quad w(x, t)$ and $p_{9}(x, t)$.

$$
w(0, t)=e^{-3 t} ; \quad w(1, t)=e^{-1-3 t} ; \quad w(x, 0)=e^{-x}
$$

The solution is $w(x, t)=e^{-x-3 t}$ if $p(t)=1+t$

First step: approximates $f(x, t)=w_{x}(x, t)-w_{t}(x, t)$

We take the base $\psi_{i}(m)=m^{i-1} e^{-m} \quad i=1,2, \cdots, 5$

Accuracy is $\left(\int_{a_{1}}^{b_{1}} \int_{a_{2}}^{b_{2}}\left|f(x, t)-p_{5}(x, t)\right|^{2} \mathrm{~d} t \mathrm{~d} x\right)^{\frac{1}{2}}=0.160504$.

In Figure 3(a) the exact solution and the approximate solution are compared Second step: approximates $w(x, t)$

We take the base $\psi_{i j}(m, z)=m^{i-1} z^{j-1} e^{-m-z} \quad i=1,2,3 \quad j=1,2,3$

Accuracy is $\left(\int_{a_{1}}^{b_{1}} \int_{a_{2}}^{b_{2}}\left|w(x, t)-p_{9}(x, t)\right|^{2} \mathrm{~d} t \mathrm{~d} x=\right)^{\frac{1}{2}}=0.0388971$.

In Figure $3(\mathrm{~b})$ the exact solution and the approximate solution are compared.

\section{Conclusions}

An equation in parabolic partial derivatives of the form $w_{t}-\left(w_{x}\right)_{x}=r(x, t)$ where the unknown function $w(x, t)$ is defined in $E=\left(a_{1}, b_{1}\right) \times\left(a_{2}, \infty\right)$ under the conditions 


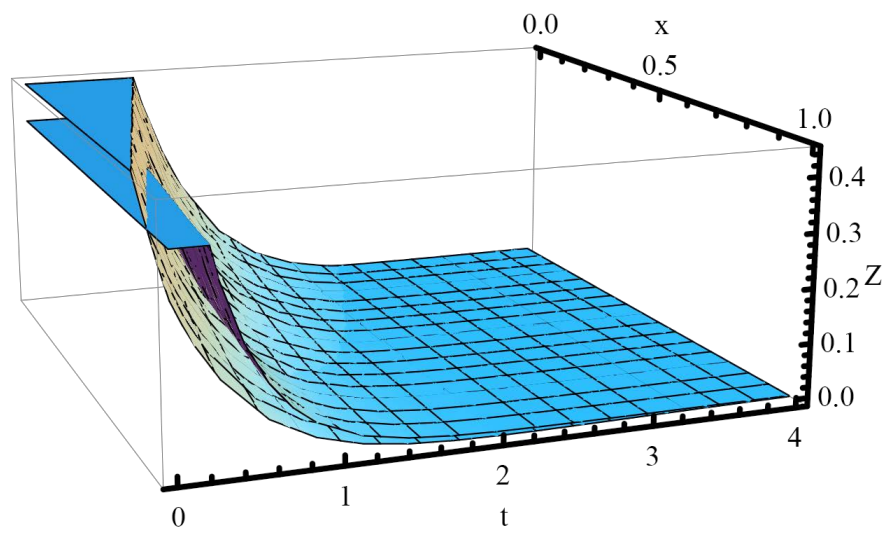

(a)

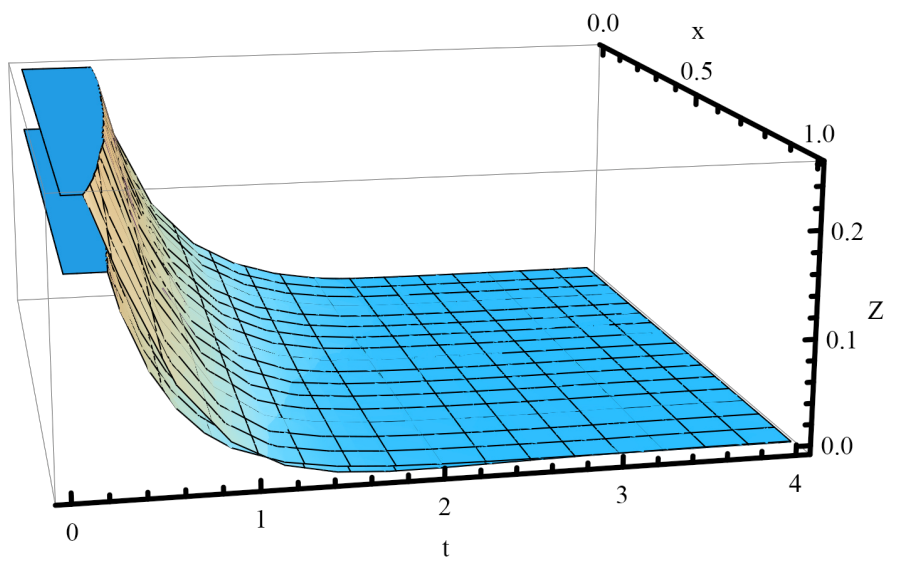

(b)

Figure 3. (a) $w_{x}(x, t)-w_{t}(x, t)$ and $p_{5}(x, t) ;(\mathrm{b}) \quad w(x, t)$ and $p_{9}(x, t)$.

$$
w\left(a_{1}, t\right)=k_{1}(t) \quad w\left(b_{1}, t\right)=k_{2}(t) \quad w\left(x, a_{2}\right)=h_{1}(t)
$$

can be solved numerically by applying inverse moments problem techniques in two steps: first consider the integral equation

$$
\int_{a_{1}}^{b_{1}} \int_{a_{2}}^{b_{2}} e^{-m(x+t)}\left(w_{x}(x, t)-w_{t}(x, t)\right) \mathrm{d} t \mathrm{~d} x=\varphi_{1}(m)
$$

and we can solve it numerically as an inverse moments problem, and get an approximate solution for $w_{x}(x, t)-w_{t}(x, t)$. Then in a second step we consider the integral equation

$$
\int_{a_{1}}^{b_{1}} \int_{a_{2}}^{b_{2}} e^{-(m+1)(x+1)-(z+1)(t+1)}(w(x, t)(t-x)) \mathrm{d} t \mathrm{~d} x=\varphi_{2}(m, z)
$$

and again we can solve it numerically as an inverse moments problem, and get an approximate solution for $w(x, t)$. It is observed that the function $r(x, t)$ is not used in calculations, but it is implicitly considered in the boundary conditions.

In this way it would be possible to solve, for example, the problem of finding $w(x, t)$ that satisfies

$$
w_{t}-\left(w_{x}\right)_{x}=p(t) w(x, t)+\Phi(x, t) \text { in } E=(0,1) \times(0, \infty)
$$


under the conditions

$$
w(0, t)=k_{1}(t) \quad w(1, t)=k_{2}(t) \quad w(x, 0)=h_{1}(t)
$$

with unknown $p(t)$ and known $\Phi(x, t)$.

\section{Acknowledgements}

We thank the Editor and the referee for their comments.

\section{References}

[1] Pintarelli, M.B. and Vericat, F. (2014) Partial Differential Equations as Three-Dimensional Inverse Problem of Moments. Journal of Mathematics and System Science, 4, 657-666.

[2] Pintarelli, M.B. (2016) Parabolic Partial Differential Equations as Inverse Moments Problem. Applied Mathematics, 7, 77-99. https://doi.org/10.4236/am.2016.71007

[3] Cherniha, R.M. (2001) New Exact Solutions of One Nonlinear Equation in Mathematical Biology and Their Properties. Ukrainian Mathematical Journal, 53, 393-411. https://doi.org/10.1023/A:1015252112419

[4] Mittal, R.C. and Jiwari, R. (2011) A Higher Order Numerical Scheme for Some Nonlinear Differential Equations: Models in Biology. International Journal for Computational Methods in Engineering Science and Mechanics, 12, 134-140. https://doi.org/10.1080/15502287.2011.564265

[5] Forsythe, G.E. and Wasow, W.R. (1960) Finite Difference Methods for Partial Differential Equations. John Wiley and Sons, New York.

[6] Zafarullah, A. (1971) Some Stable Implicit Difference Methods for Heat Equation with Derivative Boundary Condition. The Computer Journal, 14, 309-311. https://doi.org/10.1093/comjnl/14.3.309

[7] Keast, P. and Mitchell, A.R. (1966) On the Instability of the Crank Nicholson Formula under Derivative Boundary Conditions. The Computer Journal, 9, 110-114. https://doi.org/10.1093/comjnl/9.1.110

[8] Ames, W.F. (1992) Numerical Methods for Partial Differential Equations. Academic Press, Inc., New York.

[9] Lapidus, L. and Pinder, G.F. (2011) Numerical Solution of Partial Differential Equations in Science and Engineering. John Wiley and Sons, New York.

[10] Friedman, A. (2013) Partial Differential Equations of Parabolic Type, Courier Corporation.

[11] Knabner, P. and Angerman, L. (2006) Numerical Methods for Elliptic and Parabolic Partial Differential Equations. Springer, Berlin.

[12] Johnson, C. (2009) Numerical Solution of Partial Differential Equations by the Finite Element Meth. Dover Publications, Inc., New York.

[13] Nayroles, B., Touzot, G. and Villon, P. (1992) Generalizing the Finite Element Method: Diffuse Approximation and Diffuse Elements. Computational Mechanics, 10, 307-318. https://doi.org/10.1007/BF00364252

[14] Babuska, I. and Melenk, J. (1997) The Partition of Unity Method. International Journal for Numerical Methods in Engineering, 40, 727-758. https://doi.org/10.1002/(SICI)1097-0207(19970228)40:4<727::AID-NME86>3.0.CO; $\underline{2-\mathrm{N}}$

[15] Belytschko, T., Lu, Y.Y. and Gu, L. (1994) Element-Free Galerkin Methods. International Journal for Numerical Methods in Engineering, 37, 229-256. 
https://doi.org/10.1002/nme.1620370205

[16] Liu, W., Jun, S. and Zhang, Y. (1995) Reproducing Kernel Particle Methods. International Journal for Numerical Methods in Fluids, 20, 1081-1106. https://doi.org/10.1002/fld.1650200824

[17] Onate, E., Idelsohn, S., Zienkiewicz, O.C. and Taylor, R.L. (1996) A Finite Point Method in Computational Mechanics, Application to Convective Transport and Fluid Flow. International Journal for Numerical Methods in Engineering, 39, 3839-3866.

https://doi.org/10.1002/(SICI)1097-0207(19961130)39:22<3839::AID-NME27>3.0.C $\underline{\mathrm{O} ; 2-\mathrm{R}}$

[18] Atluri, S.N. and Zhu, T. (1998) New Meshless Local Petrov-Galerkin (MLPG) Approach in Computational Mechanics. Computational Mechanics, 22, 117-127. https://doi.org/10.1007/s004660050346

[19] Tataria, M. and Dehghanb, M. (2009) On the Solution of the Non-Local Parabolic Partial Differential Equations via Radial Basis Functions. Applied Mathematical Modelling, 33, 1729-1738. https://doi.org/10.1016/j.apm.2008.03.006

[20] Liszka, T. (1984) An Interpolation Method for an Irregular Net of Nodes. International Journal for Numerical Methods in Engineering, 20, 1599-1612. https://doi.org/10.1002/nme.1620200905

[21] Akheizer, N.I. (1965) The Classical Moment Problem. Olivier and Boyd, Edinburgh.

[22] Akheizer, N.I. and Krein, M.G. (1962) Some Questions in the Theory of Moment. American Mathematical Society, Providence.

[23] Shohat, J.A. and Tamarkin, J.D. (1943) The Problem of Moments. American Mathematical Society, Mathematical Surveys and Monographs, Providence. https://doi.org/10.1090/surv/001

[24] Talenti, G. (1987) Recovering a Function from a Finite Number of Moments. Inverse Problems, 3, 501-517. https://doi.org/10.1088/0266-5611/3/3/016

[25] Ang, D.D., Gorenflo, R., Le, V.K. and Trong, D.D. (2002) Moment Theory and Some Inverse Problems in Potential Theory and Heat Conduction. Lectures Notes in Mathematics, Springer, Berlin. https://doi.org/10.1007/b84019

[26] Pintarelli, M.B. and Vericat, F. (2008) Stability Theorem and Inversion Algorithm for a Generalized Moment Problem. Far East Journal of Mathematical Sciences, 30, 253-274.

[27] Pintarelli, M.B. and Vericat, F. (2011) Bi-Dimensional Inverse Moment Problems. Far East Journal of Mathematical Sciences, 54, 1-23.

[28] Pintarelli, M.B. (2015) Linear Partial Differential Equations of First Order as Bi-Dimensional Inverse Moment Problem. Applied Mathematics, 6, 979-989. https://doi.org/10.4236/am.2015.66090 
Submit or recommend next manuscript to SCIRP and we will provide best service for you:

Accepting pre-submission inquiries through Email, Facebook, LinkedIn, Twitter, etc. A wide selection of journals (inclusive of 9 subjects, more than 200 journals)

Providing 24-hour high-quality service

User-friendly online submission system

Fair and swift peer-review system

Efficient typesetting and proofreading procedure

Display of the result of downloads and visits, as well as the number of cited articles Maximum dissemination of your research work

Submit your manuscript at: http://papersubmission.scirp.org/

Or contact am@scirp.org 